\title{
Efeitos Genéticos Aditivos Direto e Materno sobre o Peso à Desmama em Animais Mestiços Europeu-Zebu
}

\author{
Kepler Euclides Filho ${ }^{1,2}$, Geraldo Ramos de Figueiredo ${ }^{1}$, Rafael Geraldo de Oliveira Alves ${ }^{1}$, \\ Luiz Otávio Campos da Silva1,2
}

\begin{abstract}
RESUMO - Os dados usados neste estudo são resultados de avaliações de cruzamentos entre as raças européias e a raça Nelore. As informações referentes a pesos à desmama de 883 animais, oriundos de seis grupos de acasalamentos envolvendo cruzamentos das raças Simental, Charolês e Chianina com a raça Nelore, retrocruzamentos e Nelore puro, foram usadas. Os pesos à desmama foram ajustados para as idades do bezerro e da mãe e para grupo contemporâneo (constituído pela combinação de ano e estação de nascimento e sexo), a fim de se estimarem os efeitos genéticos direto e materno sobre o peso à desmama. Estes efeitos foram estimados pela regressão do peso à desmama sobre a composição racial do indivíduo, que foi considerada como a proporção do genótipo do Nelore. As estimativas para os efeitos aditivos diretos foram 4,43 kg para a raça Simental, 6,58 kg para a raça Chianina e 15,24 kg para a raça Charolês. As estimativas para os efeitos maternos, entretanto, foram $41,78 \mathrm{~kg}$ para a raça Simental, $26,50 \mathrm{~kg}$ para a raça Charolês e 26,11 kg para a raça Chianina.
\end{abstract}

Palavras-chave: bovino de corte, Charolês, Chianina, Nelore, Simental, cruzamento, raça continental

\section{Direct and Maternal Genetic Additive Effects on Weaning Weight of Crossbred European-Zebu Animals}

\begin{abstract}
The data used in this study are results of the evaluations of crosses among European breeds and Nellore breed. The data regarding to the weaning weight of 883 calves resulted from six mating groups involving crosses of Simental, Charolais and Chianina breeds with Nellore, backcrosses and pure Nellore, were used. The weaning weights were adjusted for cow and calf ages and contemporary group (formed by the combination of year and season of birth and sex) to estimate direct and maternal genetic additive effects on weaning weight. These effects were estimated by regression of the weaning weight on the individual breed composition, which was considered as a proportion of Nellore genotype. The direct additive effect estimates were $4.43 \mathrm{~kg}$ for Simental breed, $6.58 \mathrm{~kg}$ for Chianina breed and $15.24 \mathrm{~kg}$ for Charolais breed. The maternal additive effect estimates, however, were $41.78 \mathrm{~kg}$ for the Simental breed, $26.50 \mathrm{~kg}$ for the Charolais breed and $26.11 \mathrm{~kg}$ for the Chianina breed.
\end{abstract}

Key Words: beef cattle, Charolais, Chianina, Nellore, Simmental, cross breeding, continental breed

\section{Introdução}

O aumento da produtividade em bovino de corte tem sido alcançado pela combinação de melhores manejo e nutrição associados à utilização de animais de maior potencial genético. Nesse sentido, os cruzamentos vêm recebendo atenção especial não só pela sua contribuição direta para o aumento da produtividade, mas também pela sua capacidade de viabilizar o ajuste do binômio genótipo-ambiente, que se constitui em componente essencial para aumento de eficiência de qualquer sistema de produção de gado de corte.

Com o incremento da utilização dos cruzamentos nos sistemas de produção de gado de corte, várias raças de origem européia têm sido introduzidas para acasalamentos, principalmente, com a raça Nelore.
Como conseqüência, tem-se a produção de matrizes mestiças de diversas raças e com diferentes "grausde-sangue" europeu-zebu. Nesse contexto, é importante ressaltar a contribuição que essas fêmeas mestiças têm trazido para aumento da produção (EUCLIDES FILHO, 1996), como conseqüência, principalmente, da influência do efeito materno sobre grande número de características de importância econômica, especialmente sobre aquelas relacionadas com o desempenho até a desmama.

Além disso, os cruzamentos têm tido grande influência na avaliação genética, principalmente, em função do incremento no uso de animais mestiços, promovendo evolução da mesma, para a denominada avaliação multiracial . Nesse sentido, de acordo com RODRIGUEZ-ALMEIDA et al. (1997), a falta de 
informações que possibilitem a partição adequada dos efeitos aditivos direto e materno, em populações multiraciais, quando da estimação de Diferenças Esperadas nas Progênies (DEP) para comparações de animais entre raças, pode resultar em ordenamento incorreto desses animais. De fato, de acordo com ARNOLD et. al. (1992), considerando que as diferenças raciais se constituem em componentes de tais DEPs, os ordenamentos dos animais estariam viesados.

Assim, o objetivo principal deste trabalho foi avaliar a importância dos efeitos aditivo direto e materno sobre o peso à desmama de animais Nelore, mestiços F1s oriundos de cruzamentos entre as raças Simental, Chianina e Charolês com a Nelore, e os provenientes de retrocruzamentos do Nelore com as fêmeas F1s.

\section{Material e Métodos}

Este estudo foi conduzido no Centro Nacional de Pesquisa de Gado de Corte (CNPGC) da Empresa Brasileira de Pesquisa Agropecuária (EMBRAPA) situado em Campo Grande, MS. A área experimental estava localizada em solos do tipo Latossolo álico fase cerradão, e as pastagens eram predominantemente constituídas por Brachiaria decumbens $c v$. Basilisk. Os animais receberam mistura mineral completa, à vontade, em cochos cobertos, sendo esta a única suplementação alimentar oferecida.

A cada dois meses os animais foram pesados. Os controles contra carrapatos e bernes foram realizados sempre que necessários, e a everminação seguiu a estratégia recomendada pelo CNPGC.

Os dados utilizados referem-se ao peso à desmama de 883 animais nascidos entre 1989 e 1991, sendo 97 Nelore $(\mathrm{N} \times \mathrm{N}), 133$ 1/2Simental - 1/2 Nelore (S x N), 136 1/2 Chianina - 1/2 Nelore (Ci x N), 115 1/2 Charolês - 1/2 Nelore (C x N), 136 3/4 Nelore - 1/4 Simental (N x SN), 122 3/4 Nelore - 1/4 Chianina ( $\mathrm{N}$ x CiN), e 144 3/4 Nelore - 1/4 Charolês ( $\mathrm{N}$ x CN).

Como os dados disponíveis eram provenientes de vacas e bezerros cujas constituições genéticas eram formadas por proporções variáveis da raça Nelore, e assumindo-se que os componentes materno e direto se combinam de forma aditiva, utilizou-se, para análise, o modelo básico proposto por KOGER et al. (1975) $\left(\mathrm{Y}=\mathrm{b}_{0}+\mathrm{A}_{\mathrm{o}} \mathrm{X}_{1}+\mathrm{A}_{\mathrm{m}} \mathrm{X}_{2}\right)$, ao qual foram incorporados os efeitos fixos de grupo contemporâneo (constituído pela combinação de ano e estação de nascimento e sexo) e idades do bezerro e da mãe como covariáveis. Assim, o modelo matemático utilizado foi o seguinte:
$\mathrm{Y}==\mathrm{b}_{0}+\mathrm{A}_{\mathrm{o}} \mathrm{X}_{1}+\mathrm{A}_{\mathrm{m}} \mathrm{X}_{2}+\mathrm{GC}_{\mathrm{i}}+\mathrm{IB}_{\mathrm{j}}+\mathrm{IV}_{\mathrm{k}}+\mathrm{E}_{\text {rro }}$ em que $\mathrm{Y}$ representa a média do grupo genético e $\mathrm{b}_{0}$, a média de quadrados mínimos de determinada característica; os subescritos "o" e "m" representam os componentes direto e materno, respectivamente; as variáveis $\mathrm{X}_{1}$ e $\mathrm{X}_{2}$ são medidas das composições raciais tomadas como proporções da raça Nelore para bezerro e vaca, respectivamente; $A_{o}$ e $A_{m}$ são os coeficientes de regressão de $\mathrm{Y}$ (peso à desmama) sobre os $\mathrm{X}_{\mathrm{i}}$ respectivos, ou seja, os efeitos dos componentes direto e materno, respectivamente; e GC representa os grupos contemporâneos e IB e IV as idades do bezerro e vaca, respectivamente.

Como os cruzamentos envolveram diferentes raças e representaram somente indivíduos Nelore, "meiosangue" e retrocruzados, optou-se pela análise das raças em separado, ou seja, foram analisados três grupos formados pela raça Nelore e cada uma das raças européias.

\section{Resultados e Discussão}

O peso à desmama, característica influenciada tanto pelo genótipo do bezerro quanto pelo efeito materno, associado ao fato de ser influenciado pela heterose, torna-se medida de desempenho extremamente importante no estabelecimento de programas de cruzamento. Apesar de a partição do peso à desmama em seus componentes heteróticos ser de grande importância, o conhecimento das diferenças em efeitos aditivos, direto e materno, para as diversas raças, assume papel também fundamental no delineamento de sistemas de cruzamentos. Isto ocorre não só pelo fato de o peso à desmama ser o principal produto da fase de cria, mas também porque o conhecimento das diferenças entre raças para efeitos diretos e maternos possibilita a escolha correta da melhor utilização e/ou da melhor combinação dessas diferenças. Esse é, sem dúvida, um elemento que contribui efetivamente para que os cruzamentos se constituam na forma mais eficiente de se obter progresso rápido nessa característica.

De modo geral, dados provenientes de campo ou de experimentos não-delineados, com o objetivo específico de se avaliar a importância do vigor híbrido, dificilmente possibilitam a obtenção de estimativas de heterose. Isso é mais evidente em situações que envolvem grande número de raças; nesses casos, há necessidade de grande número de cruzamentos envolvendo diversas raças.

No presente trabalho, os efeitos de heterose 
direta e materna estão, até certo ponto, confundidos com os efeitos aditivos direto e materno e, por isso, não puderam ser separados. Dessa forma, podem estar contribuindo para alguns vieses nas estimativas dos coeficientes. Os valores de $\mathrm{b}_{0}$ ajustados para os efeitos de grupo contemporâneo e idades do bezerro e da vaca foram 163,$52 ; 163,54$; e 158,79 para os grupos compostos pelas raças Simental, Charolês e Chianina, respectivamente. Pela semelhança entre os $\mathrm{b}_{0}$ obtidos, pode-se inferir que as diferenças existentes entre os grupos foram oriundas de diferenças observadas nas estimativas dos efeitos.

As estimativas encontradas para efeito aditivo da raça Nelore foram significativamente menores $(\mathrm{P}<0,05)$ que as observadas para as demais raças estudadas (Tabela 1). Este resultado era esperado porque todas as outras raças avaliadas são de grande porte e, portanto, com maior potencial genético para crescimento. Vale ressaltar, contudo, a grande superioridade observada para o efeito aditivo direto na raça Charolês, o que reflete o grande potencial dessa raça continental. Apesar de, no presente trabalho, ter sido observado que a raça Simental resultou em maior efeito direto que a Nelore, é importante ressaltar que essa foi a menor diferença verificada, o que correspondeu a, aproximadamente, $67 \%$ da verificada para a raça Chianina e a $29 \%$ da observada para a raça Charolês.

Da mesma forma que para os efeitos aditivos diretos, os efeitos aditivos maternos foram significativamente menores $(\mathrm{P}<0,05)$ na raça Nelore, não havendo, todavia, diferença entre os efeitos maternos para as raças Charolês e Chianina.

Possivelmente, essa superioridade observada para a raça Simental foi conseqüência de seu maior potencial de produção de leite, o que resultou em melhor

Tabela 1 - Estimativas dos efeitos aditivo direto $\left(A_{o}\right)$ e materno $\left(A_{m}\right)$ para peso à desmama, de acordo com o grupo genético

Table 1 - Additive direct (Ao) and additive maternal (Am) effect estimates for weaning weight, according to genetic group

\begin{tabular}{lcc}
\hline $\begin{array}{l}\text { Grupo genético } \\
\text { Genetic group }\end{array}$ & $\begin{array}{c}\text { Efeito direto }\left(\mathrm{A}_{\mathrm{o}}\right) \\
\text { Direct effect }\end{array}$ & $\begin{array}{c}\text { Efeito materno }\left(\mathrm{A}_{\mathrm{m}}\right) \\
\text { Maternal effect }\end{array}$ \\
$\mathrm{SxN}$ & 4,43 & \\
$\mathrm{CxN}$ & 15,24 & \\
$\mathrm{CixN}$ & 6,58 & 41,78 \\
$\mathrm{NxSN}$ & & 26,50 \\
$\mathrm{NxCN}$ & 26,11 \\
$\mathrm{NxCiN}$ & & \\
${ }^{1}$ Raças: $\mathrm{N}=$ Nelore, $\mathrm{S}=$ Simental, $\mathrm{C}=$ Charolês, $\mathrm{Ci}=$ Chianina. \\
Breeds: $\mathrm{N}=$ Nellore, $\mathrm{S}=$ Simmental, $\mathrm{C}=$ Charolais, $\mathrm{Ci}=$ Chianina.
\end{tabular}

ambiente materno para sua progênie. Esta superioridade de raças com maior potencial de produção de leite foi também observada por DIJK (1983), que trabalhou com Angus, Brown Swiss e F1s. Nesse caso, o autor observou que o efeito aditivo materno foi muito menor para o Angus. Todavia, mesmo para o efeito direto, foi verificado superioridade do Brown Swiss.

$\mathrm{Na}$ Tabela 2 são apresentados os pesos ajustados à desmama de acordo com o grupo genético do bezerro. Este ajustamento foi feito aplicando-se as estimativas dos efeitos aditivos direto e materno obtidos ( Tabela 1) na equação $\mathrm{Y}=\mathrm{b}_{0}+\mathrm{A}_{\mathrm{o}} \mathrm{X}_{1}+$ $\mathrm{A}_{\mathrm{m}} \mathrm{X}_{2}$. Por exemplo, a equação para o retrocruzamento de fêmeas $1 / 2$ Simental - $1 / 2$ Nelore com touros Nelore $(\mathrm{N} \mathrm{x} \mathrm{SN})$ foi $188=163,52+0,75(4,43)+0,5(41,78)$. A superioridade das mães "meio-sangue" é resultado não só da maior produção de leite normalmente verificada nessas fêmeas quando comparadas com raças puras, mas também é fruto dessa mesma heterose para outros componentes da habilidade materna, bem como do ambiente permanente.

MACNEIL et al. (1982), estudando a influência dos efeitos direto e materno sobre o peso à desmama, em bezerros oriundos de diversas raças, também observaram que o efeito aditivo materno da raça Simental foi maior que o verificado para as outras raças estudadas. EUCLIDES FILHO et al. (1998) observaram que vacas "meio-sangue" Simental produziram bezerros mais pesados, à desmama, que as F1s Chianina-Nelore. Estes pesos, no entanto, não foram diferentes dos observados para progênie de vacas F1s Charolês-Nelore. A importância do efeito aditivo materno foi também ressaltada por DILLARD et al. (1980). Esses autores, trabalhando com animais oriundos das raças Angus, Hereford e Charolês, e seus

Tabela 2 - Médias de quadrados mínimos para peso à desmama para os diferentes grupos de acasalamento

Table 2 - $\quad$ Least square means for weaning weight according to different mating groups

\begin{tabular}{lcc}
\hline $\begin{array}{l}\text { Grupo genético } \\
\text { Mating group }\end{array}$ & $\begin{array}{c}\text { Número de animais } \\
\text { Number of animals }\end{array}$ & $\begin{array}{c}\text { Peso à desmama } \\
\text { Weaning weight }\end{array}$ \\
\hline $\mathrm{N} \times \mathrm{N}$ & 97 & 158 \\
$\mathrm{~S} \times \mathrm{N}$ & 133 & 166 \\
$\mathrm{CxN}$ & 136 & 171 \\
$\mathrm{CixN}$ & 115 & 162 \\
$\mathrm{~N} x \mathrm{SN}$ & 136 & 188 \\
$\mathrm{~N} x \mathrm{CN}$ & 122 & 188 \\
$\mathrm{NxCiN}$ & 144 & 177 \\
\hline
\end{tabular}

${ }^{1}$ Raças: $\mathrm{N}=$ Nelore, $\mathrm{S}=$ Simental, $\mathrm{C}=$ Charolês, $\mathrm{Ci}=$ Chianina. Breeds: $N=$ Nellore, $S=$ Simmental, $C=$ Charolais, $C i=$ Chianina. 
cruzamentos, verificaram superioridade do Charolês quanto à habilidade materna. Na verdade, o Charolês, à semelhança do que foi observado neste trabalho, sobrepujou as demais raças também para o efeito aditivo direto para as características estudadas.

Superioridade, para peso à desmama, de filhos mestiços oriundos de touros Simental comparados aos de touros Charolês foi, todavia, verificada por NOTTER et al. (1978).

Importantes efeitos aditivos direto e materno foram também observados, para a raça Simental e Gelbvieh, por RODRIGUEZ-ALMEIDA et al. (1997). Efeitos aditivos diretos positivos e de alta magnitude para raças continentais foram ainda observados por PEACOCK et al. (1981). Esses autores verificaram valores negativos dos coeficientes estimados para bezerros filhos de Brahman, os quais foram atribuídos ao baixo desempenho ponderal desses animais associado ao grande efeito heterótico observado nos mestiços de Brahman.

\section{Conclusões}

Considerando-se as condições em que foi desenvolvido o estudo, as estimativas de efeito aditivo materno indicam que sistemas de cruzamentos com o objetivo principal de produzir de bezerros desmamados produzem, potencialmente, mais utilizando fêmeas $1 / 2$ Simental - $1 / 2$ Nelore do que aqueles com fêmeas $1 / 2$ Charolês - $1 / 2$ Nelore, ou $1 / 2$ Chianina $1 / 2$ Nelore, enquanto as estimativas de efeitos aditivos diretos indicam o uso de touros Charolês para produção de bezerros "meio-sangue". O uso de touros Charolês sobre fêmeas $1 / 2$ Simental - $1 / 2$ Nelore poderia otimizar a utilização dos efeitos genéticos aditivo direto e materno em sistema de produção de gado de corte.

\section{Referências Bibliográficas}

ARNOLD, J. W., BERTRAND, J. K., BENYSHEK, L. L. 1992. Animal model for genetic evaluation of multibreed data. $J$. Anim. Sci., 70(11):3322-3333.

DIJK, A. V. Additive breed and heterosis effects among crosses of Brown Swiss and Angus breeds. [S. L.]: University of Florida, 1983. 52 p. Thesis (Master of Science) - University of Florida, 1983.

DILlARD, E. U., RODRIGUEZ, O., ROBISON, O. W. 1980. Estimation of additive and nonadditive direct and maternal genetic effects from crossbreeding beef cattle. J. Anim. Sci., 50(4):653-663.

EUCLIDES FILHO, K. 1996. O melhoramento genético e os cruzamentos em bovinos de corte. Campo Grande: EMBRAPACNPGC, 35p. (EMBRAPA-CNPGC. Documentos, 63).

EUCLIDES FILHO, K., FIGUEIREDO, G. R. de, SILVA, L. O. C. da et al. 1998. Idade aos $165 \mathrm{~kg}$ de peso vivo para progênies de Nelore, Fleckvieh, Chianina, Charolês, F1s e Retrocruzas. R. Bras. Zootec., 27(5):1-7.

KOGER, M., PEACOCK, F. M., KIRK, W. G. et al. 1975. Heterosis effects on weaning performance of BrahmanShorthorn calves. J. Anim. Sci., 40(5):826-833.

MACNEIL, M. D., DINKEL, C. A., VAN VLECK, L. D. 1982. Individual and maternal additive and heterotic effects on 205day weight in beef cattle. J. Anim. Sci., v. 54, n. 5, p. 951-656.

NOTTER, D. R., CUNDIFF, L. V., SMITH, G. M. et al. 1978. Characterization of biological types of cattle. XII. Milk production in young cows and transmitted and maternal effects on preweaning growth of progeny. J. Anim. Sci., 46(5):903-912.

PEACOCK, F. M., KOGER, M., OLSON, T. A. et al. 1981. Additive genetic and heterosis effects in crosses among cattle breeds of british, european and zebu origin. J. Anim. Sci., 52(5):1007-1013.

RODRIGUEZ-ALMEIDA, F. A., VAN VLECK, L. D., GREGORY, K. E. 1997. Estimation of direct and maternal breed effects for prediction of expected progeny differences for birth and weaning weights in three multibreed populations. J. Anim. Sci., 75(5):1203-1212.

Recebido em: 14/04/98

Aceito em: 09/09/98 